Article

\title{
Pulsed Ultraviolet Light Reduces Immunoglobulin E Binding to Atlantic White Shrimp (Litopenaeus setiferus) Extract
}

\author{
Sandra Shriver ${ }^{1}$, Wade Yang ${ }^{1, *}$, Si-Yin Chung ${ }^{2}$ and Susan Percival ${ }^{1}$
}

1 Department of Food Science \& Human Nutrition, University of Florida, P.O. Box 110370, 359 FSHN Bldg. Newell Drive, Gainesville, FL 32611, USA;

E-Mails: sandrakshriver@ufl.edu (S.S.); percival@ufl.edu (S.P.)

2 Southern Regional Research Center, Agricultural Research Service, U.S. Department of Agriculture, 1100 Robert E. Lee Blvd., Bldg 001 SRRC, New Orleans, LA 70124, USA; E-Mail: siyin.chung@ars.usda.gov

* Author to whom correspondence should be addressed; E-Mail: wade.yang@ufl.edu; Tel.: +1-352-392-1991 ext. 507; Fax: +1-352-392-9467.

Received: 18 May 2011 / Accepted: 19 June 2011 / Published: 24 June 2011

\begin{abstract}
Pulsed ultraviolet light (PUV), a novel food processing and preservation technology, has been shown to reduce allergen levels in peanut and soybean samples. In this study, the efficacy of using PUV to reduce the reactivity of the major shrimp allergen, tropomyosin (36-kDa), and to attenuate immunoglobulin $\mathrm{E}$ (IgE) binding to shrimp extract was examined. Atlantic white shrimp (Litopenaeus setiferus) extract was treated with PUV (3 pulses/s, $10 \mathrm{~cm}$ from light source) for $4 \mathrm{~min}$. Tropomyosin was compared in the untreated, boiled, PUV-treated and [boiled+PUV]-treated samples, and changes in the tropomyosin levels were determined by sodium dodecyl sulfate-polyacrylamide gel electrophoresis (SDS-PAGE). IgE binding of the treated extract was analyzed via immunoblot and enzyme-linked immunosorbent assay (ELISA) using pooled human plasma containing IgE antibodies against shrimp allergens. Results showed that levels of tropomyosin and IgE binding were reduced following PUV treatment. However, boiling increased IgE binding, while PUV treatment could offset the increased allergen reactivity caused by boiling. In conclusion, PUV treatment reduced the reactivity of the major shrimp allergen, tropomyosin, and decreased the IgE binding capacity of the shrimp extract.
\end{abstract}


Keywords: allergen; allergy; shrimp; pulsed ultraviolet light; PUV, tropomyosin; $\operatorname{IgE}$ antibodies

\section{Introduction}

In the United States, approximately $6 \%$ of children and $3.7 \%$ of adults are affected by one or more food allergies. Eight major food sources, including cow's milk, shellfish, egg, fish, tree nuts, peanuts, soybean, and wheat, account for approximately $85 \%$ of all food allergies [1]. Allergies to shellfish, such as shrimp, affect $0.1 \%$ of American children and $2 \%$ of American adults, making shellfish allergies the most common type of food allergy in adults [2].

The major heat-stable allergen of shrimp is a $36-\mathrm{kDa}$ protein known as tropomyosin, also referred to as Sa-II or Pen a 1 [3-5]. Tropomyosin plays an important role in muscular contraction, as well as in the regulation of cellular structure and motility [6]. Although present in both vertebrates and invertebrates, tropomyosin is known to elicit an allergic reaction only when it is derived from invertebrate sources, such as crustaceans, arachnids, insects and mollusks [5]. Two additional shrimp allergens have been identified: myosin light chain (20-kDa) [7] and arginine kinase (40-kDa) [8-10]. However, studies suggest that the majority of shrimp allergenicity can be attributed to tropomyosin protein alone $[3,5,11]$, where tropomyosin was recognized by $82 \%$ of patients with shrimp allergies and was shown to inhibit IgE binding to whole-body shrimp extract in $85-95 \%$ of patients.

According to Jeong and others [6], the most frequent symptoms of shrimp-induced allergies include itching, hives, swelling of the lips and tongue, pulmonary symptoms, gastrointestinal symptoms, and anaphylactic shock. Methods such as oral and sublingual immunotherapy have been used in attempt to prevent shrimp-induced allergies, yet the only completely effective approach to date is total avoidance [12]. Complete avoidance is often difficult and inconvenient, considering the prevalence of food allergens in a multitude of products. Thus, researchers are seeking diverse ways, such as postharvest treatment methods, to reduce the allergen reactivity of food products before they reach the consumer. When an allergen enters the body, immunoglobulin $\mathrm{E}$ ( $\mathrm{IgE}$ ) antibodies elicit an immune response by binding to specific epitopes on an allergen, which can be linear or conformational [13]. Postharvest methods have the potential to alter these epitopes by disrupting or masking amino acid sequences (e.g., protein fragmentation or genetic modification) or by altering the conformation of the protein (e.g., protein denaturation, protein crosslinking, or aggregation). Postharvest methods, including power ultrasound [14], gamma irradiation [15], and high hydrostatic pressure processing [16], have been shown to alter allergen reactivity by modifying allergen structure. More recently, pulsed ultraviolet light (PUV), an emerging technology, has been employed to reduce the allergen reactivity of peanut products [17,18] and soy extracts [19].

Pulsed ultraviolet light is considered more effective in food processing (specifically microbial inactivation) than conventional, or continuous, UV light, because of its instantaneous high-energy pulses and greater capability to penetrate. In a PUV system, electrical energy is captured and stored in a capacitor and is ultimately released in short pulses as ultraviolet, infrared, and visible light (approximately 54\%, 20\%, and 26\%, respectively) [20]. The resultant bursts can be several thousand 
times more intense than continuous UV light [21]. Upon coming into contact with a sample, the light interacts with molecules, which are excited and-upon returning to ground state-liberate energy as photons or heat, which can induce chemical changes. Thus the efficacy of PUV has been attributed to photochemical, photothermal, and photophysical reactions [21]. These effects may contribute to changes in protein structure and reduction in IgE binding to allergens.

At a shorter exposure (e.g., seconds), PUV is normally regarded as nonthermal, as the temperature rise of food is insignificant. However, at a longer exposure (e.g., minutes), PUV can generate significant photothermal effects and incur considerable temperature rise and moisture loss to the sample [17-19,22-25]. It has also been found that prolonged UV light treatment caused formation of insoluble complexes in food, depolymerization of starch, peroxidation of unsaturated fatty acids, carbohydrate crosslinking, protein crosslinking, and protein fragmentation [26-29]. The significant photothermal effect of PUV after an extended exposure enables the PUV technology to be applied directly to solid foods, e.g., whole almond [23], for allergen mitigation. Li [23] exposed the whole almond kernels to PUV for 4-7 min. It was found the whole almond treated with PUV for 4 min had a desirable and pleasurable roasted almond flavor and taste, and its $\operatorname{IgE}$ biding capacity was pronouncedly reduced, although the exact mechanism of PUV interactons with the solids is still unknown.

Based on the studies of PUV treatment on peanut and soybean allergens [17-19], we hypothesized that PUV treatment of Atlantic white shrimp extract would alter the reactivity of the major shrimp allergen, tropomyosin, and consequently reduce the overall allergenic potential of the shrimp extract. Our objective was to examine the efficacy of PUV exposure on the inactivation of major shrimp allergen by measuring changes in tropomyosin level and IgE binding.

\section{Experimental Section}

\subsection{Materials}

Frozen Atlantic white shrimp (Litopenaeus setiferus) were purchased de-headed and shelled from Publix Supermarkets, Inc. (Lakeland, FL). Coomassie Plus (Bradford) Protein assay, bovine serum albumin (BSA), StartingBlock/Tris-buffered saline/Tween-20 (StartingBlock), GelCode Blue gel staining reagent, $o$-phenylenediamine dihydrochloride (OPD) and SuperSignal West Pico Chemiluminescent (ECL) substrate were purchased from Thermo Fisher Scientific Inc. (Rockford, IL). Electrophoresis equipment and reagents including pre-cast Tris-HCL minigels (4-15\%), Mini-PROTEAN $^{\circledR}$ Tetra cell tanks, Laemmli sample buffer, Tris-glycine transfer buffer, Tris-glycine-SDS running buffer, nitrocellulose membrane $(0.45 \mu \mathrm{m})$ and Trans-blot SD Semi-dry Transfer cells were purchased from Bio-Rad Laboratories, Inc. (Hercules, CA). Pooled human plasma from 3 patients with history of shrimp allergy was obtained from PlasmaLabs International (Everett, WA) for immunoblotting and enzyme-linked immunosorbent assay (ELISA). The plasma-specific IgE level for pooled human plasma with antibodies specific to shrimp was measured by the ImmunoCAP method performed by PlasmaLabs and was determined to be $92 \mathrm{kU} \mathrm{L}^{-1}$. A secondary-detection antibody, mouse anti-human IgE conjugated to horseradish peroxidase (HRP), was obtained from Invitrogen (Carlsbad, CA). Rat monoclonal anti-tropomyosin (IgG isotype) and rabbit polyclonal anti-rat IgG-H\&L conjugated to HRP antibodies were purchased from Abcam Inc. (Cambridge, MA). 
Costar Enzyme Immunoassay (EIA) polystyrene 96-well plates (Corning, NY) and Immobilon P blotting polyvinylidene fluoride (PVDF) membrane $(0.45 \mu \mathrm{m})$ (Millipore Corporation, Bedford, MA) were used for immunological assays.

\subsection{Preparation of Atlantic White Shrimp Crude Protein Extract}

Shrimp extract was prepared following the methods of Motoyama and others [30,31] with modification. Briefly, shrimp (25 g) was ground in a food processor at low speed for $10 \mathrm{~s}$. A volume of $0.6 \mathrm{M} \mathrm{KCl}$ in $0.01 \mathrm{M}$ phosphate buffer $(200 \mathrm{~mL}, \mathrm{pH}$ 7) was added to the processed tissue, and the mixture was homogenized at the high-speed setting $(10,000 \mathrm{rpm})$ for $1 \mathrm{~min}$ using a BioSpec BioHomogenizer (Bartlesville, OK). The buffer was carefully selected in order to solubilize the major allergen while maintaining its native state. Protein concentration was measured with Bradford assay using BSA protein standards. The extract was diluted to a concentration of $5 \mathrm{mg} / \mathrm{mL}$ with $0.6 \mathrm{M} \mathrm{KCl}$ in $0.01 \mathrm{M}$ phosphate buffer ( $\mathrm{pH} 7$ ). Samples were treated immediately or stored packaged on ice in a styrofoam container placed at $4{ }^{\circ} \mathrm{C}$ for no longer than one week.

\subsection{Preparation of Boiled Shrimp Extract}

A common way of preparing shrimp is by boiling, but literature shows the allergen immunoactivity of shrimp is not reduced in general or in some cases is even increased by boiling [32-34]. Consumption of boiled or steamed shrimp resulted in more severe allergy skin test responses in some patients than raw shrimp [33,34]. Liu and others [35] reported that although iELISA demonstrated that the raw shrimp extracts had higher IgE binding than the boiled shrimp extracts, dot-blot results showed higher IgE binding to the purified tropmyosin from boiled shrimp than raw shrimp. In this study, boiled shrimp extracts were also prepared by heating in boiling water for $4 \mathrm{~min}$ and then tested for its IgE binding capacity, which was compared to the PUV treated samples. A volume of $10 \mathrm{~mL}$ of crude shrimp protein extract was placed in boiling water for $4 \mathrm{~min}$ in a loosely capped $15 \mathrm{~mL}$ centrifuge tube. Following heat treatment, samples were cooled on ice, and protein concentration was measured.

\subsection{Treatment of Shrimp Extract with PUV}

Raw and boiled samples $(10 \mathrm{~mL}, 5 \mathrm{mg} / \mathrm{mL})$ were treated with a Xenon Steripulse-XL RS-3000 batch PUV sterilization unit (Wilmington, MA), as described by Chung and others [17], to examine the effect of PUV on the immunoreactivity of both raw and boiled shrimp samples. The treatment of boiling followed by PUV was to exemine if synergistic or antagonistic effect on allergen reactivity existed between boiling and PUV treatments.

Pulses were emitted at a rate of $3 / \mathrm{s}$ with a pulse width of $360 \mu \mathrm{s}$, and samples were positioned at a distance of $10 \mathrm{~cm}$ from the quartz window of the PUV lamp in aluminum dishes with a diameter of $7.2 \mathrm{~cm}$. Under these conditions, the maximum energy level of the emitted radiation was $0.27 \mathrm{~J} / \mathrm{cm}^{2}$ per pulse as per the factory calibration. Temperature of the samples was recorded using an Omega OS423-LS non-contact infrared thermometer (Omega Engineering, Inc., Stamford, CT), and the samples were cooled on ice following treatment. Changes in volume were measured, and protein concentration was determined with Bradford assay. 


\subsection{Electrophoresis of Treated Shrimp Extract}

Samples were analyzed under reducing conditions as described by Laemmli [36]. Briefly, a sample containing protein $(12 \mu \mathrm{g})$ was combined with sample buffer $(62.5 \mathrm{mM}$ Tris-HCL, pH 6.8, 2\% SDS, $25 \%$ glycerol, $0.01 \%$ bromophenol blue, $0.05 \% \beta$-mercaptoethanol) in a microcentrifuge tube. The mixture was heated in boiling water for $5 \mathrm{~min}$. The sample was then subjected to electrophoresis within a $4-15 \%$ Tris-glycine gel for $1.5 \mathrm{~h}$ at $150 \mathrm{~V}$ per the manufacturer's recommendations. Subsequently, the gel was stained with GelCode Blue reagent for $2 \mathrm{~h}$ and destained for $1 \mathrm{~h}$ with deionized water. The protein bands were scanned with a Canon Pixma MP160 scanner.

\subsection{Determination of IgE- and IgG-Binding to Tropomyosin with Western Blot}

Following electrophoresis, proteins were transferred onto a PVDF blotting membrane at $15 \mathrm{~V}$ for 30 min. Nonspecific binding sites were blocked for $1 \mathrm{~h}$ at room temperature (RT) with StartingBlock blocking buffer. The membrane was incubated overnight at $4{ }^{\circ} \mathrm{C}$ with pooled human plasma containing anti-shrimp IgE antibodies (1:80) diluted in blocking buffer. After washing with Tris buffered saline containing $0.1 \%$ Tween 20 (TBST), the blot was then incubated in mouse anti-human IgE-HRP diluted 1:1,000 in blocking buffer for $1 \mathrm{~h}$ at RT. The blot was again washed, and incubated in SuperSignal West Pico Chemiluminescent substrate for $5 \mathrm{~min}$ at RT. The protein bands were developed on an $\mathrm{X}$-ray film. To visualize changes in tropomyosin band intensity, the above procedure was replicated, replacing the primary antibody with rat monoclonal anti-tropomyosin $(\operatorname{IgG})(1: 1,000)$ and the secondary antibody with rabbit polyclonal anti-rat IgG-HRP $(1: 40,000)$.

\subsection{Determination of IgE Binding to Treated Shrimp Extract with Dot Blot}

A nitrocellulose membrane was blotted with 1.25 and $2.5 \mu \mathrm{g}$ of raw, boiled, PUV-treated, and [boiled+PUV]-treated shrimp extract protein and allowed to dry at $4{ }^{\circ} \mathrm{C}$. Following blocking with StartingBlock blocking buffer, the blot was incubated overnight at $4{ }^{\circ} \mathrm{C}$ in a pooled human plasma containing anti-shrimp IgE antibodies (1:80) diluted in blocking buffer. The blot was washed and then incubated in mouse anti-human IgE-HRP diluted 1:1,000 in blocking buffer (1 h; RT). The blot was again washed, and incubated in SuperSignal West Pico Chemiluminescent substrate for 5 min at RT. The protein spots were developed on an x-ray film.

\subsection{Determination of IgE Binding to Treated Shrimp Extracts with Indirect ELISA}

Polystyrene 96-well plates were coated overnight at $4{ }^{\circ} \mathrm{C}$ with untreated, boiled, PUV-treated, and [boiled+PUV]-treated shrimp extract diluted in phosphate-buffered saline (PBS) to a concentration of $20 \mu \mathrm{g} / \mathrm{mL}$ (100 $\mu \mathrm{L}$ per well in triplicates). The plates were subsequently washed with TBST and blocked with StartingBlock blocking buffer $(200 \mu \mathrm{L}$ per well) at room temperature for $2-3 \mathrm{~h}$. Pooled human plasma containing IgE antibodies specific for shrimp allergens was diluted in PBS (1:10) and added in equal amounts to each well $(100 \mu \mathrm{L})$. The plate was incubated at room temperature with gentle shaking for $1 \mathrm{~h}$. After washing with TBST, each well was then incubated with secondary antibody, monoclonal mouse anti-human $\operatorname{IgE}$ conjugated to $\operatorname{HRP}(1: 3,000)$, for $1 \mathrm{~h}(100 \mu \mathrm{L}$ per well) with gentle shaking. The wells were again washed, and an OPD substrate $(0.5 \mathrm{mg} / \mathrm{mL})$ dissolved in 
$0.1 \mathrm{M}$ citrate buffer ( $\mathrm{pH} 5.5$ ) and $0.03 \%$ hydrogen peroxide was added to each well (100 $\mu \mathrm{L}$ per well). The reaction was stopped at 15-30 min with $2.5 \mathrm{~N}$ sulfuric acid (100 $\mu \mathrm{L}$ per well), and absorbance was measured at $490 \mathrm{~nm}$ using a Spectramax $340^{384}$ spectrophotometer (Molecular Devices, Inc. Sunnyvale, CA).

\subsection{Statistical Analysis}

Statistical analysis was conducted using one-way analysis of variance (ANOVA) with the SAS 9.2 software package (Cary, N.C.). Significant differences $(\alpha=0.05)$ between means of the untreated (control) and treated (boiled, PUV-treated, and [boiled+PUV]-treated) samples for total IgE binding were determined using least significant difference (LSD) and Duncan's Multiple Range tests.

\section{Results and Discussion}

\subsection{Optimal PUV Treatment Time for Shrimp Extract}

To establish an appropriate treatment time, several time courses for PUV treatment were tested. SDS-PAGE and Western blot analyses were used to determine the minimum exposure time at which PUV treatment led to a reduction in both tropomyosin level and IgE binding. An SDS-PAGE analysis (Figure 1) illustrated that tropomyosin bands $(36-\mathrm{kDa})$ remained in the extract following PUV treatments of 1,2, and 3 min, but were reduced in samples treated for 4-6 min. Densitometry analysis verified the reduction in tropomyosin band intensity at 4-6 min. It is noted that Figure 1 also shows other two minor allergenic proteins $(16.5-\mathrm{kDa}$ and $20-\mathrm{kDa})$, which have also been reported in other studies [37].

Figure 1. An SDS-PAGE profile of shrimp extract treated with PUV at $0,1,2,3,4,5$, and 6 min. Molecular weight marker $(\mathrm{M})$ is shown. The band corresponding to tropomyosin $(36-\mathrm{kDa})$ is highlighted with an arrow.

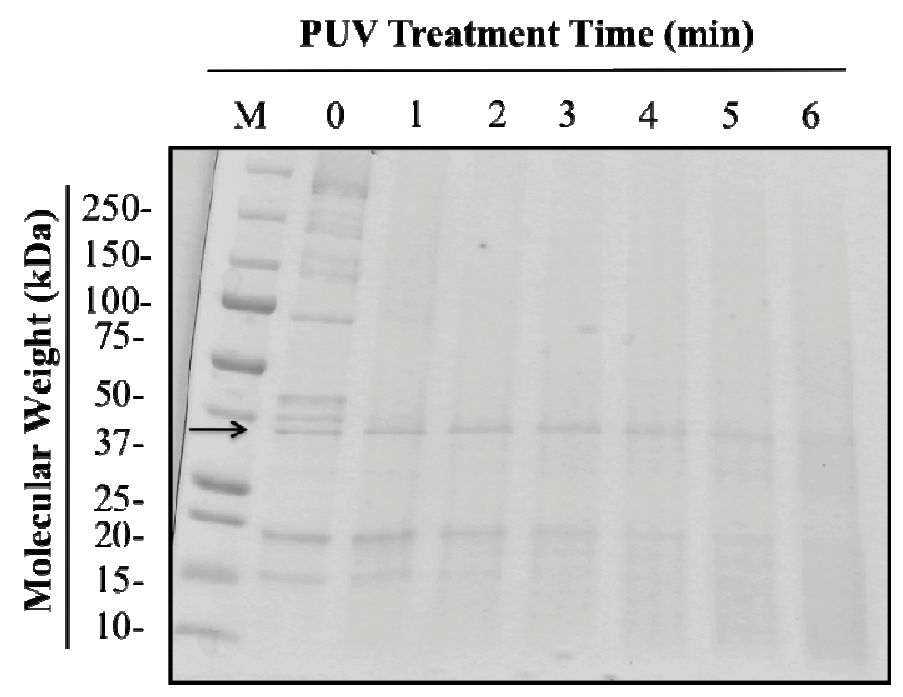

Western blot analysis of the PUV-treated extracts at 0-6 min (Figure 2) showed that IgE binding to tropomyosin was markedly reduced at 4-6 $\mathrm{min}$, compared to 0-3 min. Because patterns between 
4 and 6 min were similar and a higher moisture loss was observed at $6 \mathrm{~min}, 4 \mathrm{~min}$ was chosen as the optimal treatment time for subsequent experimentation.

Figure 2. Western blot analysis of shrimp extract samples treated with PUV at 0 (control), $1,2,3,4,5$, and 6 min using pooled human plasma from 3 individuals containing $\operatorname{IgE}$ antibodies against shrimp. Tropomyosin bands (36-kDa) are highlighted within a box.

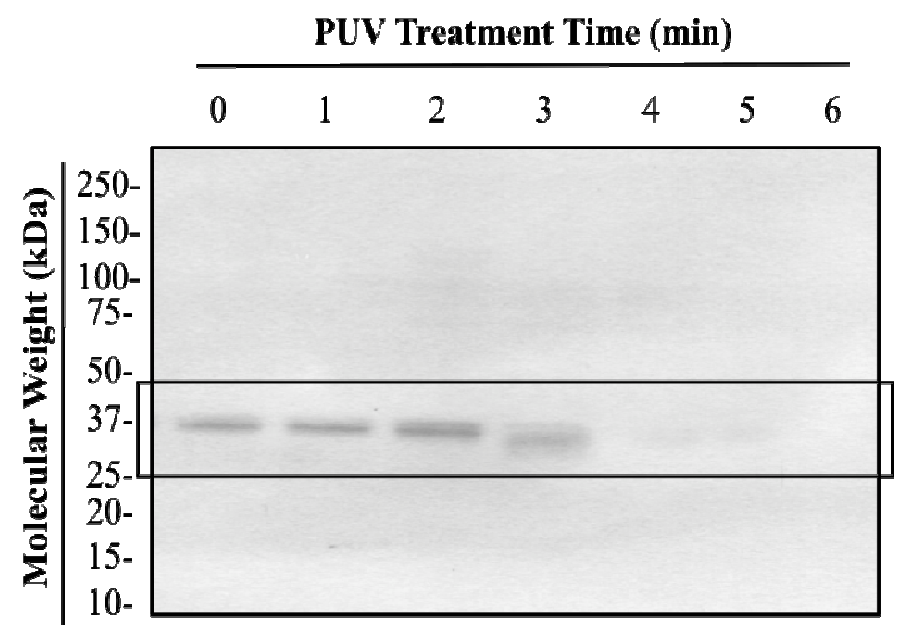

3.2. Changes in Tropomyosin Band Intensity of Untreated, Boiled, PUV-Treated, and [Boiled+PUV]-Treated Shrimp Extracts

As illustrated in the SDS-PAGE profile (Figure 3), a 36-kDa band representing tropomyosin was present following treatments with boiling, PUV, and boiling+PUV. A decrease in tropomyosin was observed in both PUV- and [boiled+PUV]-treated samples (lanes 3 and 4), whereas the boiled extract (lane 2) did not show a change in tropomyosin density compared to the control. These results were verified using densitometry analysis.

Figure 3. An SDS-PAGE profile of untreated (1), boiled (2), PUV-treated (3), and [boiled+PUV]-treated (4) shrimp extracts. Molecular weight marker is shown (M). An arrow highlights the bands corresponding to tropomyosin $(36-\mathrm{kDa})$.

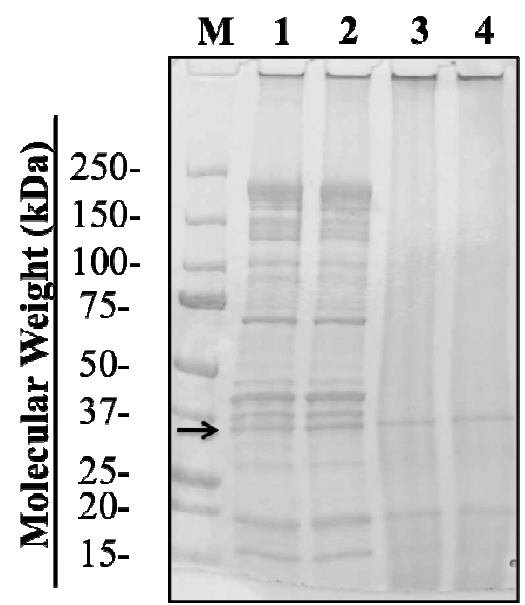


Like Figure 1, Figure 3 also shows the behavior of other two allergenic proteins (16.5-kDa and $20-\mathrm{kDa}$ ) under boiling and PUV treatments. The $20-\mathrm{kDa}$ protein followed a similar trend to tropomyosin's in terms of boiling and PUV effects, while boiling alone did not change much the 16.5-kDa protein, but PUV caused it to be almost undetectable as illustrated on lanes 3 and 4 .

Resistance to thermal denaturation or degradation is characteristic of the tropomyosin protein, as it is recognized for its heat stability [3]. However, instantaneous pulses of energy generated during prolonged PUV treatment may cause more intense localized heating [38,39], which may contribute to the reduction of tropomyosin. On the other hand, UV exposure, which is basically nonthermal, can also cause protein crosslinking or fragmentation [27,29]. So, we believe both the nonthermal and photothermal effects of PUV played a role in reducing the allergenic reactivity of shrimp proteins in this study.

\subsection{IgE- and IgG-binding to Untreated, Boiled, PUV-treated, and [Boiled+PUV]-Treated Shrimp Extracts}

\subsubsection{Western Blot}

The four treated samples were analyzed with Western blot using IgE antibodies against shrimp, to evaluate allergen reactivity, and an anti-tropomyosin antibody (IgG), to evaluate tropomyosin levels. Figure 4 demonstrates a notable decrease in IgE binding and tropomyosin levels following PUV treatment, as evidenced by decreases in band intensity. The marked difference between the control and PUV treatment further supports the SDS-PAGE data (Figure 1) that tropomyosin levels were indeed reduced at $4 \mathrm{~min}$. Boiling, however, appeared to increase IgE binding, based on densitometry analysis of the control versus boiled.

Figure 4. Western blots using (a) pooled human plasma containing IgE antibodies against shrimp and (b) monoclonal anti-tropomyosin antibody (IgG) to analyze untreated (1), boiled (2), PUV-treated (3) and [boiled+PUV]-treated (4) shrimp extracts. Tropomyosin (36-kDa) is highlighted using a box.

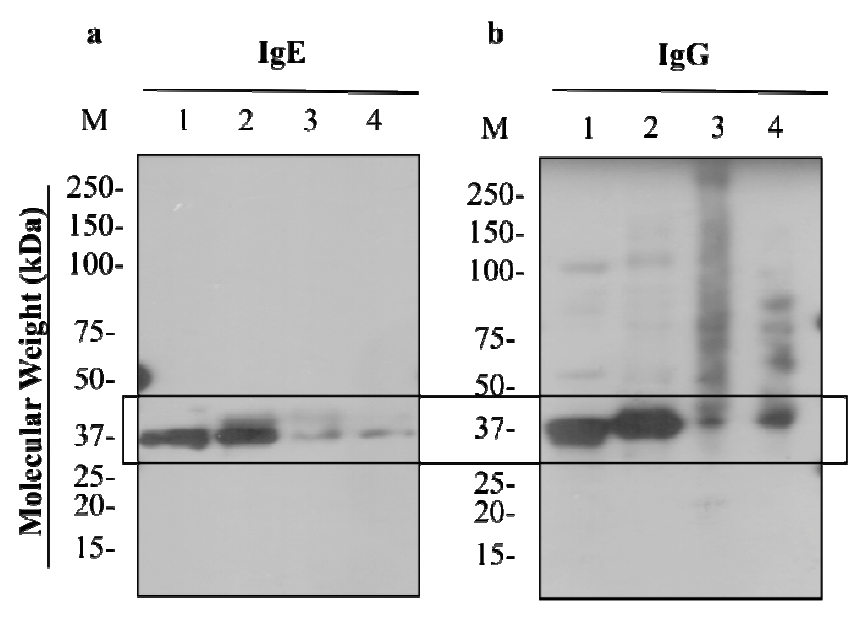

Presently, it is still unknown exactly how the IgE binding was reduced by PUV radiation, but it is believed to relate to localized photochemical, photothermal and photophysical effects of PUV, which, 
as mentioned earlier, can cause protein modifications, including protein fragmentation, denaturation, or crosslinking and affect IgE binding. Fragmented protein sections that are smaller than the resolution limit of the gel may travel through the acrylamide pores more quickly, causing them to ultimately be lost into the chamber buffer. Conversely, proteins that have been modified by crosslinking are often too large to migrate through the gel or migrate with slight difficulty, thereby resulting in a smeared appearance in SDS-PAGE and Western blots. Chung and others [40] have demonstrated the presence of protein smears due to the crosslinking of peanut allergens caused by enzymatic reactions. Also, protein band smearing has been observed in the allergens of peanuts that have been heated or roasted [41]. While band smearing was not detected in the blot with $\operatorname{IgE}$ (Figure 4a), they can be seen in blots using an anti-tropomyosin antibody. As shown in Figure 4b, tropomyosin bands and others were notably smeared in PUV-treated samples, but not in boiled and control samples. This finding indicates that modification or crosslinking of tropomyosin might have occurred during the PUV treatment.

Additionally, Taheri-Kafrani and others [42] have illustrated the smearing of milk allergens that have been modified via the Maillard reaction, which is a non-enzymatic browning or protein-carbohydrate reaction. Several studies [42-44] have linked glycation or Maillard reaction to the modification of allergens and their subsequent changes in IgE binding. A study on squid tropomyosin [44] found that $\operatorname{IgE}$ binding was suppressed with the progression of the Maillard reaction. However, in one study using scallop tropomyosin [43], an increase in the allergen potency was displayed with the progression of Maillard reaction. Whether Maillard reaction could occur in the shrimp extract during PUV treatment is not known. Considering the high energy produced by PUV and the instantaneous heat absorbed by the molecules [39] (i.e., proteins/carbohydrate in the shrimp extracts), it is possible that a Maillard reaction may occur, but such a postulation needs to be further investigated.

\subsubsection{Dot Blot}

Dot blot analysis was performed to determine the allergen reactivity of the shrimp protein extract as a whole-that is, tropomyosin and other possible allergens that were not detected via Western blot analysis. IgE binding to whole shrimp extract was greatly reduced following PUV treatment (Figure 5). However, there was an increase in IgE binding following boiling treatment. Interestingly, PUV appeared to attenuate or negate the boiling effect, as shown in the [boiled+PUV]-treated sample; IgE binding was notably reduced compared to the boiled-only sample. Of all the treatments, PUV alone displayed the most reduction in IgE binding.

The finding that boiling or heating can lead to an increase in IgE binding (Figure 5) is not unusual, because the effects of thermal processing on food allergens have been studied extensively, and, in different studies, heating has been shown to either decrease or increase allergen potency. For example, one study [41] described a 90-fold increase in $\operatorname{IgE}$ binding of roasted peanuts over raw peanuts, whereas another study reported that roasting actually decreased the overall allergen reactivity of hazelnuts [45]. It has also been noted that children with milk allergies show a tolerance to extensively heated milk [46]. In this dot blot analysis (Figure 5), boiling caused an increase in IgE binding possibly not just due to tropomyosin itself, but also due to other proteins present in the whole extract. These proteins could include arginine kinase $(40-\mathrm{kDa})[8,9]$ and myosin light chain $(20-\mathrm{kDa})[7,38]$ which have both been shown to play a minor role in shrimp allergy. Also, the minor $16.5 \mathrm{kDa}$ protein was also persistent during the boiling treatment (Figure 3). The effects of boiling on shrimp reactivity 
are consistent with the results of Carnes and others [32] who also found that boiled shrimp extracts were more immunoreactive in both in vivo skin prick trials and in vitro direct ELISA results.

Figure 5. Dot blot analysis of untreated, boiled, PUV-treated, and [boiled+PUV]-treated shrimp extract using pooled human plasma containing $\operatorname{IgE}$ antibodies against shrimp.

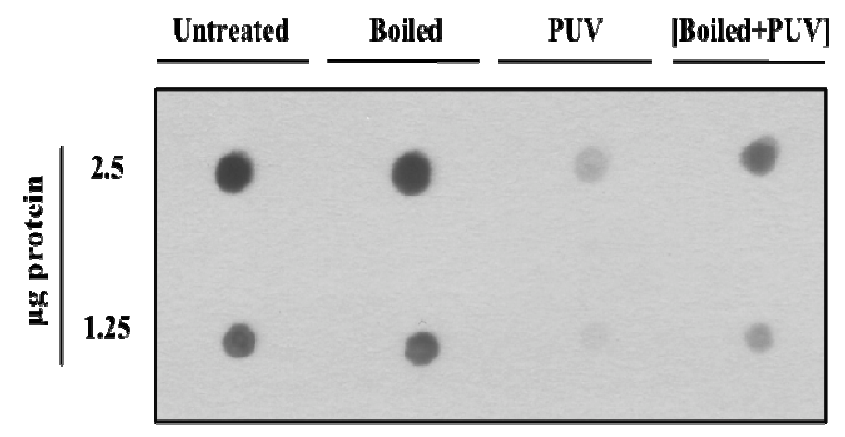

\subsubsection{Indirect ELISA}

To support the dot blot data in Figure 4, IgE binding of the four treated shrimp extracts was also determined using an indirect ELISA. Again, a significant decrease $(\alpha=0.05)$ in IgE binding was seen in the PUV-treated extract, compared to the control (Figure 6). An increase in IgE binding was observed in the boiled extract while there was no significant change $(\alpha=0.05)$ in IgE binding of the [boiled+PUV]-treated extract, as compared to the control. The finding was in agreement with the data of dot blot (Figure 4).

Figure 6. Indirect ELISA illustrating changes in IgE binding compared to untreated, boiled, PUV-treated, and [boiled+PUV]-treated shrimp extracts using pooled human plasma containing IgE antibodies against shrimp. $\mathrm{A}=$ absorbance of the sample; $\mathrm{A}_{0}=$ absorbance of untreated sample. Data are expressed as mean $\pm \operatorname{SEM}(n=5)$. Results are relative values, normalized to the untreated sample; untreated is standardized and set to 1 . Values that are significantly different $(\alpha=0.05)$ from the untreated sample are annotated as **.

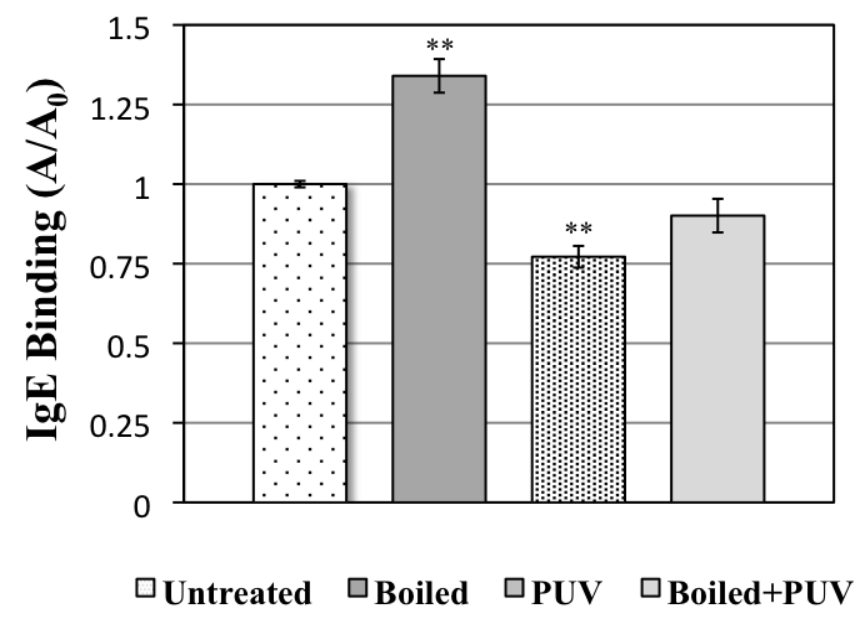


Reduction in IgE binding of PUV-treated extract was likely explained by changes in the amount of detectable tropomyosin described above in SDS-PAGE (Figures 1 and 3) and Western blot analysis (Figures 2 and 4). In the [boiled+PUV]-treated sample, IgE binding did not appear to change, compared to the control. This was because tropomyosin increased after boilng but was offset to the control level after the PUV treatment. That is to say, the increase and decrease in IgE binding, respectively, due to boiling and PUV treatment, may negate each other, thus resulting in a negligible change in IgE binding of the [boiled+PUV] extract. Such an effect may be deemed as an antagonistic effect.

\subsection{Temperature and Volume Changes Following Treatment of Shrimp Extract}

As mentioned earlier, PUV is considered a nonthermal method when used for brief periods of time (several seconds); however, following a PUV treatment period of $4 \mathrm{~min}$, a sample surface temperature of $68.3 \pm 2.5{ }^{\circ} \mathrm{C}$ was detected using an infrared thermometer immediately after the PUV pulses stopped and the treatment chamber door was opened. It must be noted that there was a $5-10 \mathrm{~s}$ delay in probing the sample surface temperature, while the instantaneous temperatures of the sample during the PUV treatment could be higher. Previous trials using thermocouples to monitor temperature during PUV treatment were unsuccessful, because extended PUV exposure of the metal probe confounded the readings. Fiber optic temperature sensing may be a way to go for recording accurately the sample temperature during PUV treatment, which was not conducted in this study. For shorter PUV treatments, a type-K thermocouple produced by Omega Engineering, Inc. (Stanford, CT), has been utilized [47] for temperature measurement without confounding a complication in temperature reading. A study that analyzed PUV treatment for decontamination of shell-eggs [47] reported an increased surface temperature of $10.5 \pm 1.2^{\circ} \mathrm{C}$ after $30 \mathrm{~s}$ at $9.5 \mathrm{~cm}$ distance from the quartz window of the PUV lamp.

Following boiling, PUV, and [boiling+PUV] treatments, moisture loss in each sample was measured: boiled-only $(5.83 \pm 2.3 \%)$, PUV-only $(29 \pm 3.6 \%)$, and boiled with PUV (39.7 $\pm 2.5 \%)$. Moisture loss was higher in the PUV-treated samples because the samples were not enclosed during PUV treatment (the purpose was to ensure the maximum absorption of the PUV radiation). By contrast, moisture loss was minimal in the boiled sample, because the samples were loosely capped during boiling. Chung and others [17] also noted volume reductions of approximately $40 \%$ following PUV treatment. To correct for moisture loss in the samples, protein measurements were taken after treatments, and these values were used for subsequent experiments.

Excessive moisture loss is indicative of a sample temperature during the PUV treatment that was well above the boiling point of water at atmosphere, which caused the water to evaporate. The significant temperature increases due to the photothermal effect of PUV and the capability of PUV in mitigating allergens may potentially be used in conjunction with food preparation to simultaneously heat the food and reduce its allergenic potency. Li [23] took advantage of this unique feature of PUV radiation to desirably roast the whole almond after 4-7 min exposure and yet considerably reduce its IgE biding capacity. This can also be a potential method to cook the peeled whole shrimp and significantly reduce its allergen, although this idea was not tested yet in this study. 


\section{Conclusions}

A marked decrease in IgE binding of the shrimp extract following PUV treatment has been demonstrated. The decrease was likely due to a reduction in the detectable level of tropomyosin in the PUV-treated extract as shown in SDS-PAGE (Figure 1) and Western blot (Figure 2). Furthermore, the appearance of protein band smearing, as illustrated in Figure 4, is likely due to the modification, such as crosslinking, of tropomyosin and may contribute to the decrease in $\operatorname{IgE}$ binding. Boiling increased $\operatorname{IgE}$ binding to whole shrimp sample; however, the effect of boiling was offset when it was combined with PUV treatment. Overall, PUV was found to be capable of reducing the allergenic potency of shrimp extracts. Further optimization is still needed before the PUV technology can be adopted. In vivo studies are also needed to verify the reduction in allergenic potency of the PUV-treated shrimp extracts.

\section{Acknowledgments}

The authors gratefully acknowledge Melanie Correll, University of Florida, Department of Agricultural and Biological Engineering, for her intellectual input concerning this manuscript. Publication of this article was funded in part by the University of Florida Open-Access Publishing Fund.

\section{References}

1. Ellman, L.K.; Chatchatee, P.; Sicherer, S.H.; Sampson, H.A. Food hypersensitivity in two groups of children and young adults with atopic dermatitis evaluated a decade apart. Pediatr. Allergy. Immunol. 2002, 13, 295-298.

2. Sicherer, S.H.; Sampson, H.A. Food allergy. J. Allergy. Clin. Immunol. 2010, 125, S116-125.

3. Shanti, K.N.; Martin, B.M.; Nagpal, S.; Metcalfe, D.D.; Rao, P.V., Identification of tropomyosin as the major shrimp allergen and characterization of its IgE-binding epitopes. J. Immunol. 1993, 151, 5354-5363.

4. Jeoung, B.J.; Reese, G.; Hauck, P.; Oliver, J.B.; Daul, C.B.; Lehrer, S.B. Quantification of the major brown shrimp allergen Pen a 1 (tropomyosin) by a monoclonal antibody-based sandwich ELISA. J. Allergy. Clin. Immunol. 1997, 100, 229-234.

5. Reese, G.; Ayuso, R.; Lehrer, S.B. Tropomyosin: an invertebrate pan-allergen. Int. Arch. Allergy. Immunol. 1999, 119, 247-258.

6. Jeong, K.Y.; Hong, C.S.; Yong, T.S. Allergenic tropomyosins and their cross-reactivities. Protein Pept. Lett. 2006, 13, 835-845.

7. Ayuso, R.; Grishina, G.; Bardina, L.; Carrillo, T.; Blanco, C.; Ibanez, M.D.; Sampson, H.A.; Beyer, K. Myosin light chain is a novel shrimp allergen, Lit v 3. J. Allergy. Clin. Immunol. 2008, 122, 795-802.

8. Yu, C.J.; Lin, Y.F.; Chiang, B.L.; Chow, L.P. Proteomics and immunological analysis of a novel shrimp allergen, Pen m 2. J. Immunol. 2003, 170, 445-453.

9. Garcia-Orozco, K.D.; Aispuro-Hernandez, E.; Yepiz-Plascencia, G.; Calderon-de-la-Barca, A.M.; Sotelo-Mundo, R.R. Molecular characterization of arginine kinase, an allergen from the shrimp Litopenaeus vannamei. Int. Arch. Allergy. Immunol. 2007, 144, 23-28. 
10. Shiomi, K.; Sato, Y.; Hamamoto, S.; Mita, H.; Shimakura, K. Sarcoplasmic calcium-binding protein: identification as a new allergen of the black tiger shrimp Penaeus monodon. Int. Arch. Allergy. Immunol. 2008, 146, 91-98.

11. Daul, C.B.; Slattery, M.; Reese, G.; Lehrer, S.B. Identification of the major brown shrimp (Penaeus aztecus) allergen as the muscle protein tropomyosin. Int. Arch. Allergy. Immunol. 1994, 105, 49-55.

12. Skripak, J.M.; Sampson, H.A. Towards a cure for food allergy. Curr. Opin. Immunol. 2008, 20, 690-696.

13. Tanabe, S. Epitope Peptides and Immunotherapy. Curr. Protein Pept. Sci. 2007, 8, 109-118.

14. Li, Z.; Lin, H.; Cao, L.M.; Jameel, K. Effect of high intensity ultrasound on the allergenicity of shrimp. J. Zhejiang Univ. Sci. B 2006, 7, 251-256.

15. Zhenxing, L.; Hong, L.; Limin, C.; Jamil, K. The influence of gamma irradiation on the allergenicity of shrimp (Penaeus vannamei). J. Food Eng. 2007, 79, 945-949.

16. Hildebrandt, S.; Schutte, L.; Stoyanov, S.; Hammer, G.; Steinhart, H.; Paschke, A. In vitro determination of the allergenic potential of egg white in processed meat. J. Allergy. (Cairo) 2010 , 2, 155-165.

17. Chung, S.Y.; Yang, W.; Krishnamurthy, K., Effects of pulsed UV-light on peanut allergens in extracts and liquid peanut butter. J. Food Sci. 2008, 73, C400-404.

18. Yang, W.; Mwakatage, N.R.; Goodrich-Schneider, R.; Krishnamurthy, K.; Rababah, T.M. Mitigation of major peanut allergens by pulsed ultraviolet light. Food Bioprocess Technol. 2011, DOI 10.1007/s11947-011-0615-6.

19. Yang, W.W.; Chung, S.Y.; Ajayi, O.; Krishnamurthy, K.; Konan, K.; Goodrich-Schneider, R., Use of pulsed ultraviolet light to reduce the allergenic potency of soybean extracts. J. Food Eng. 2010, 6, 1-12.

20. Shriver, S.K.; Yang, W. Thermal and nonthermal methods for allergen control. Food Eng. Rev. 2011, 3, 26-43.

21. Krishnamurthy, K.; Demirci, A.; Irudayaraj, J. M. Inactivation of Staphylococcus aureus in milk using flow-through pulsed UV-light treatment system. J. Food Sci. 2007, 72, M233-239.

22. Wakatage, N.R. Efficacy of Pulsed UV Light Treatment on Removal of Peanut Allergens. M.S. Thesis, Department of Food and Animal Sciences, Alabama A\&M University, Normal, AL, USA, 2008.

23. Li, Y. Effect of Pulsed Ultraviolet Light, High Hydrostatic Pressure and Non-thermal Plasma on the Antigenicity of Almond. M.S. Thesis, Department of Agricultural and Biological Engineering, University of Florida, Gainesville, FL, USA, 2011.

24. Nooji, J. Reduction of Wheat Allergen Potency by Pulsed Ultraviolet Light, High Hydrostactic Pressure and Nonthermal Plasma. M.S. Thesis, Department of Food Science and Human Nutrition, University of Florida, Gainesville, FL, USA, 2011.

25. Shriver, S.K. Effect of Selected Emerging Nonthermal Processing Methods on the Allergen Reactivity of ATLANTIC White Shrimp (Litopenaeus Setiferus). M.S. Thesis, Department of Food Science and Human Nutrition, University of Florida, Gainesville, FL, USA, 2011.

26. Fiedorowicz, M.; Tomasik, P.; Lii, C.Y. Degradation of starch by polarised light. Carbohydr. Polymer. 2001, 45, 79-87. 
27. Greenberg, J.R. Ultraviolet light-induced crosslinking of mRNA to proteins. Nucleic Acids Res. 1979, 6, 715-32.

28. Kramer, G.F.; Norman, H.A.; Krizek, D.T.; Mirecki, R.M. Influence of UV-B radiation on polyamines, lipid peroxidation and membrane lipids in cucumber. Phytochemistry 1991, 30, 2101-2108.

29. Friso, G.; Barbato, R.; Giacometti, G.M.; Barber, J. Degradation of D2 protein due to UV-B irradiation of the reaction centre of photosystem II. FEBS Letters 1994, 339, 217-221.

30. Motoyama, K.; Suma, Y.; Ishizaki, S.; Nagashima, Y.; Lu, Y.; Ushio, H.; Shiomi, K. Identification of tropomyosins as major allergens in Antarctic krill and mantis shrimp and their amino acid sequence characteristics. Mar. Biotechnol. 2008, 10, 709-718.

31. Motoyama, K.; Suma, Y.; Ishizaki, S.; Nagashima, Y.; Shiomi, K. Molecular cloning of tropomyosins identified as allergens in six species of crustaceans. J. Agric. Food Chem. 2007, 55, 985-991.32.Carnes, J.; Ferrer, A.; Huertas, A.J.; Andreu, C.; Larramendi, C.H.; FernandezCaldas, E. The use of raw or boiled crustacean extracts for the diagnosis of seafood allergic individuals. Ann. Allergy Asthma Immunol. 2007, 98, 349-354.

32. Carnes, J.; Ferrer, A.; Huertas, A.J.; Andreu, C.; Larramendi, C.H.; Fernandez-Caldas, E. The use of raw or boiled crustacean extracts for the diagnosis of seafood allergic individuals. Ann. Allergy Asthma Immunol. 2007, 98, 349-354.

33. Daul, C.B.; Morgan, J.E.; Hughes, J.; Lehrer, S.B. Provocation-challenge studies in shrimp-sensitive individuals. J. Allerg. Clin. Immunol. 1988, 81, 1180-1186.

34. Naqpal, S.; Rajappa, L.; Metcalfe, D.D.; Rao, P.V. Isolation and characterization of heat-stable allergens from shrimp (Penaeus indicus). J. Allerg. Clin. Immunol. 1989, 83, 26-36.

35. Liu, G.-M.; Cheng, H.; Nesbit, J.B.; Su, W.-J.; Gao, M.-J.; Maleki, S.J. Effects of Boiling on the IgE-Binding Properties of Tropomyosin of Shrimp (Litopenaeus vannamei). J. Food. Sci. 2010, 75, T1-T5.

36. Laemmli, U. K. Cleavage of structural proteins during the assembly of the head of bacteriophage T4. Nature 1970, 227, 680-685.

37. Samson, K.T.R; Chen, F.H.; Miura, K.; Odajima, Y.; Likura, Y.; Rivas, M.N.; Minoguchi, K.; Adachi, M. IgE Binding to Raw and Boiled Shrimp Proteins in Atopic and Nonatopic Patients with Adverse Reactions to Shrimp. Int. Arch. Allergy. Immunol. 2004, 133, 225-232.

38. Muranov, K.; Maloletkina, O.; Poliansky, N.; Kleymenov, S.; Rozhkov, S.; Goryunov, A.; Ostrovsky, M.; Kurganov, B. Mechanism of aggregation of UV-irradiated $\beta \mathrm{L}$-crystallin. Exper. Eye. Res. 2011, 92, 76-86.

39. Gomez-Lopez, V.; Ragaert, P.; Debevere, J.; Devlieghere, F. Pulsed light for food decontamination: a review. Trends Food Sci. Technol. 2007, 18, 464-473.

40. Chung, S.-Y.; Kato, Y.; Champagne, E.T. Polyphenol oxidase/caffeic acid may reduce the allergenic properties of peanut allergens. J. Sci. Food Agr. 2005, 2631-2637.

41. Maleki, S.J.; Chung, S.Y.; Champagne, E.T.; Raufman, J.P. The effects of roasting on the allergenic properties of peanut proteins. J. Allergy. Clin. Immunol. 2000, 106, 763-768. 
42. Taheri-Kafrani, A.; Gaudin, J.C.; Rabesona, H.; Nioi, C.; Agarwal, D.; Drouet, M.; Chobert, J.M.; Bordbar, A.K.; Haertlft, T. Effects of heating and glycation of beta-lactoglobulin on its recognition by IgE of sera from cow milk allergy patients. J. Agr. Food. Chem. 2009, 57, 4974-4982.

43. Nakamura, A.; Watanabe, K.; Ojima, T.; Ahn, D.H.; Saeki, H. Effect of maillard reaction on allergenicity of scallop tropomyosin. J. Agr. Food. Chem. 2005, 53, 7559-7564.

44. Nakamura, A.; Sasaki, F.; Watanabe, K.; Ojima, T.; Ahn, D.H.; Saeki, H. Changes in allergenicity and digestibility of squid tropomyosin during the Maillard reaction with ribose. J. Agr. Food. Chem. 2006, 54, 9529-9534.

45. Hansen, K.S.; Ballmer-Weber, B.K.; Luttkopf, D.; Skov, P.S.; Wuthrich, B.; Bindslev-Jensen, C.; Vieths, S.; Poulsen, L.K. Roasted hazelnuts--allergenic activity evaluated by double-blind, placebo-controlled food challenge. Allergy 2003, 58, 132-138.

46. Nowak-Wegrzyn, A.; Bloom, K.A.; Sicherer, S.H.; Shreffler, W.G.; Noone, S.; Wanich, N.; Sampson, H.A. Tolerance to extensively heated milk in children with cow's milk allergy. J. Allergy Clin. Immunol .2008, 122, 342-347.

47. Keklik, N. M.; Demirci, A.; Puri, V. M. Inactivation of Listeria monocytogenes on unpackaged and vacuum-packaged chicken frankfurters using pulsed UV-light. J. Food Sci. 2009, 74, M431-M439.

(C) 2011 by the authors; licensee MDPI, Basel, Switzerland. This article is an open access article distributed under the terms and conditions of the Creative Commons Attribution license (http://creativecommons.org/licenses/by/3.0/). 